\title{
Is the Privacy Paradox a Matter of Psychological Distance? An Exploratory Study of the Privacy Paradox from a Construal Level Theory Perspective
}

\author{
Ruwan Bandara \\ Faculty of Business \\ University of Wollongong \\ Australia \\ hmrjb180@uowmail.edu.au
}

\author{
Mario Fernando \\ Faculty of Business \\ University of Wollongong \\ Australia \\ mariof@uow.edu.au
}

\author{
Shahriar Akter \\ Faculty of Business \\ University of Wollongong \\ Australia \\ sakter@uow.edu.au
}

\begin{abstract}
Institutional operators in the digital marketplace have delighted consumers with precise, highly personalized and customized products and services through the collection and mining of customers' personally identifiable data. However, the ethical conduct of online businesses continues to be a debatable issue, due to the increasing concerns over information privacy. Despite such controversies, scrutiny of consumer behavior has shown that consumers' concerns for privacy do not transfer into protective behaviors or abstinence during online activity. The aim of this study is to illuminate the disparity known as the 'privacy paradox' through the directions of the construal level theory. Based on semistructured interviews with 21 online shopping consumers, we explain that, due to spatial, temporal, social, and hypothetical distance of privacy values, privacy is construed as an abstract phenomenon influencing the formation of distant-future attitudes and intentions rather than actual behavior.
\end{abstract}

\section{Introduction}

The extraordinary technological developments in recent years have transformed the modus operandi of human thinking and behavior. The dawn of big data analytics, mobile devices and services, ubiquitous and wireless connectivity, and Internet of Things are among many other advancements that tether humantechnology concurrence. The impact of these technologies on business and consumption has changed the fundamental dynamics of markets leading to a global digital economy. With enticing personalized and customized services, convenience, ease of use and several other advantages, online shopping continues to thrive in the current digital marketplace [1].
The consumer-generated data have become relentlessly important in today's digital environment for its ability to generate massive revenues [2] and deliver competitive advantage [3] for businesses. Hence, the collection, storing, transmission, and mining of personally identifiable information has escalated in recent years [4].

While such trends have lured consumers to enjoy greater service quality and benefit from highly customized and personalized services, they have also attracted debate over information privacy and security. According to recent privacy reports, in US, $45 \%$ consumers are more worried about their privacy than one year ago [5] and in Australia 69\% are more concerned about their privacy than five years ago [6].

In the face of numerous benefits in online shopping and distressing threats to privacy, consumers are prompted to make a value judgment or a trade-off, especially about information disclosure. Interestingly, research highlights the fact that, despite consumers' highly stated privacy concerns, they do not take adequate measures to protect their sensitive information. Neither do they refrain from disclosing their information [7, 8]. This phenomenon, where consumers' actual behavior differs from their stated privacy concerns, is known as the privacy paradox [7, 9]. Due to its implications for e-commerce, egovernment, online social networking, and privacy regulations, several efforts have been undertaken to unravel the privacy paradox. Nevertheless, it is still considered "a complex phenomenon that requires extensive further research" [9, p. 122].

Construal Level Theory (CLT) rests on the ability of the human mind to mentally represent any object or event at different levels, based on how near or distant they appear from one's immediate reality $[10,11]$. Psychologically distant objects or events are mentally construed at higher levels, characterized by their abstract and superordinate features, while psychologically proximal objects are construed more 
concretely, recognized by their peripheral and secondary features [11]. For instance, construal of an action at higher level causes the action to be valued in the distant future. Individual morals and values which are abstract and superordinate in nature are identified as higher construals that are more appealing in the distant future, and that guide future judgements and behaviors [12].

Through the application of CLT, we examine the gap between consumers' privacy concerns and their actual information disclosure behavior. The broader research question we attempt to answer is this: how can level of construal and aspects of psychological distance (i.e. spatial, temporal, social, and hypothetical) explain the privacy paradox of consumers in the online shopping context? This exploratory study is based on the perceptions gathered through 21 semi-structured interviews from online shopping consumers.

\section{Research context}

Significant innovations and technological developments in Australia during the last decade transformed existing business models and industry structures to blend in with the global movement towards the 'digital'. Australian consumers have demonstrated great appetite for online consumption, whereby $62 \%$ of internet users make online purchases and $74 \%$ of users conduct online banking and financial activities. This has led Australian businesses to generate massive income from online transactions [13]. All the same, as in most digital economies, privacy and security issues have retarded the use of online services. According to the latest government report [6], using online services has become the biggest privacy risk and $58 \%$ of consumers have decided not to deal with some businesses due to privacy and security risks. It is daunting for the businesses to learn that $69 \%$ of Australians are more worried about their privacy than they were five years ago. Although there is evidence of regulatory mechanisms and technical interventions being put in place to battle privacy issues, research on consumer privacy concerns and behavior is scarce. An in-depth review of privacy literature $[14,15]$ reveals there has been only a handful of academic research emanating from the Australian context [e.g., 16, 17, $18]$.

\section{Literature review}

\subsection{Information privacy and the privacy paradox}

Privacy has been a hot topic of interest for scholars over several decades and has been scrutinized under different lenses in several disciplines. General privacy is the overarching umbrella covering numerous dimensions of privacy, such as physical and information privacy. With the growth and widespread use of the internet and online platforms, the evolution of online information privacy can be identified [14]. Privacy has been theorized as a human right, a commodity that can be exchanged, a mental state, and as the ability to control (see [14] for a review). Throughout this paper, the term privacy is used as a reference to online information privacy and for this study we consider privacy as a right or a moral value of consumers, which is not absolute, but which varies according to the state of mind and which is exchanged for perceived benefits.

Due to the complexities of measuring privacy itself, the measurement of privacy concerns has been widely used as a proxy. The impact of privacy concerns on different behavior outcomes, including intention to transact, disclose information, and adoption and use of new technologies, has been extensively researched in the privacy scholarship [14]. Interestingly, several studies have found privacy concerns not to be a valid predictor of privacy behavior $[9,19]$. This dissension generally stems from what scholars identify as the privacy paradox of consumers, in which concerns for privacy are not reflected in, or transformed into, consumers' behavior [9].

Several attempts have been undertaken to understand the gap between privacy concerns and behavior, but only very few have endeavored clearly to explain this paradox [19]. Kokolakis [9] conducted an in-depth review of the privacy paradox literature and identified four broader areas of investigation. Privacy calculus theory-based explanations predicate that individuals perform a calculus or a trade-off between the expected benefits (i.e. of information disclosure) and potential loss of privacy [20]. When benefits are perceived to be equal or greater than the risks, individuals tend to ignore their concerns for privacy [20, 21]. Social theory-based explanations posit that individuals, as members of online communities, face a dilemma as they select between their emotional ties or attraction to these online communities and potential risk to their privacy. Studies reveal that individuals are greatly influenced by social rewards [22] and social norms [23] that could override expressed privacy concerns. Studies based on economic and social theories basically postulate that consumers engage in a cost-benefit analysis or a value-judgment between privacy values/risks and other benefits, such as gratification or social rewards. However, these studies do not necessarily discuss either the underlying 
processes or individuals' cognitive and psychological aspects of engaging in such a cost-benefit calculation or valuation.

Though limited in number, some scholars have investigated the privacy paradox phenomenon beyond economic and social theory-based explanations, using cognitive and situational aspects. Research on behavioral economics has extensively highlighted the impact of heuristics and cognitive biases on individual decision-making. Some Information System (IS) scholars have also examined aspects such as optimistic bias [24], over-confidence bias [25], affect heuristic [26], and hyperbolic discounting [27] on privacy behavior. Similarly, a limited number of studies have investigated how lack of knowledge and information asymmetries [28] can explain the privacy paradox. These cognitive and psychological aspects provide a different level of justification compared with rational cost-benefit and social-factors based interpretations. Despite the logical explanations provided by numerous theories, privacy paradox is considered a complex phenomenon which up to date has not been fully explained [9]. Therefore, we follow the directions of construal level theory to discuss how mental construals and psychological distance levels influence privacy attitudes and behaviors.

\subsection{Construal level theory (CLT) of psychological distance}

It has taken the attention of scholars from numerous disciplines to examine why individual aspects such as values, attitudes, and personality traits fail to consistently predict individual behavior [12]. Construal level theory $[10,11]$ can be deemed as useful to explain the discrepancy between someone's values, attitudes, or intentions and their actual behavior. CLT postulates the ability of the human mind to mentally represent a stimulus (e.g., object, event, person, decision) at different levels (i.e. low vs high), based on how close or distant that object is from one's immediate reality in the here and now. The higher level mental construal involves the abstract, decontextualized, and superordinate aspects of an event, whereas lower mental construals represent the concrete, contextual, and incidental details. The construal of an event is dependent upon the level of psychological distance, the "subjective experience that something is close or far away from the self, here and now', and is determined by where, when, to whom, and whether an event occurs [11, p. 440]. These factors respectively represent the aspects of psychological distance identified by the CLT: spatial, temporal, social, and hypothetical distance.
Spatial distance indicates that individuals construe faraway things or events more abstractly and nearby things more concretely [30]. Studies on temporal distance have found that individuals represent events happening in the past or future abstractly compared to events happening in the immediate environment [32]. Social distance relates to the level of personal closeness to a thing. Things related to a person or his/her in-group are mentally represented more concretely and vice versa [31]. Hypothetical distance implies that unlikely things are construed abstractly while probable things are construed concretely [32]. Accordingly, more distant future events, happening in spatially far-off places, related to other people, and unlikely to occur, are psychologically distant from our immediate experience and are therefore mentally construed at a higher level. A bi-directional relationship can be seen between construal level and distance; "more distant objects will be construed at a higher level, and high-level construal will bring to mind more distant objects"' [11, p. 444].

Several findings and contentions of CLT can be considered important to elucidate the privacy paradox. Due to their abstract, superordinate, and broadly applicable nature, moral principles and values are considered high-level construals that guide individuals' decisions and behaviors in distant situations [12]. Similarly, transgressions that occur in distant situations (e.g., in future or related to others) are considered to have moral implications more often than transgressions occurring in the immediate surrounding. Therefore, moral failures at a distance are judged more harshly [33]. When the action gets real, values become weak determinants, and incidental and situational aspects have a greater impact on behavior, which suggests values often predict distant intentions rather than actual behaviors [34]. Thus psychological distance can be identified as a significant moderator between values and mitigating circumstances. CLT studies have also found that when individuals are faced with value conflicts, central values of a person, which constitute a higher construal, guide individuals in psychologically distant situations. When the situation is more immediate, secondary values prominently influence the choices one makes [35].

Another important contention of CLT is that individuals consider the desirability of an action (i.e. why we do something) rather than the feasibility of that action (i.e. how we do something) as the psychological distance of the activity increases [29]. Research on time discounting has found the individual tendency more likely to value a near-future reward than a distant-future reward, regardless of the size of the distant-future reward [36]. CLT suggests that distance shifts the overall attractiveness of a choice more 
towards the higher-level value than the lower-value [29]. With these assertions and findings of CLT, we investigate how values received from online shopping collide with individual value of privacy which can create a paradox among online consumers.

Recently, Hallam and Zanella [19] found evidence on how temporally-near social networking rewards undermine temporally-distant privacy risks. The impact of spatial, social, and hypothetical distance on privacy behavior is yet to be explored in the literature. Literature provides limited but interesting application of CLT in the online context. Some recent studies include: impact of abstract versus concretely framed advertising messages [37]; influence of temporal and spatial distance on virtual service separability [38]; and impact of spatial distance on online distrust and reluctance to purchase [39]. The applicability of CLT in the online environment is rather useful and appropriate.

\section{Research methods}

We conducted an exploratory qualitative study to understand the cognitive aspects of privacy decision making through the firsthand knowledge of online consumers in the current digital marketplace. An exploratory design was suitable for our purpose to examine privacy behavior in detail, especially due to the unavailability of prior research on the impact of multiple dimensions of psychological distance on privacy decision making. Similarly, a qualitative approach was appropriate for the study as it helps to understand meticulously what surrounds a certain phenomenon and to capture the rich nuances of responses when compared with quantified data from large samples [40]. Given the fact that the majority of the IS studies are based on survey or laboratory based research [15], the importance of in-depth qualitative research has been highlighted [41, 42]. An exploratory qualitative study was also duly appropriate to deal with conflicting results in privacy paradox findings [9] and to move beyond the experimental nature in CLT studies.

\subsection{Data collection and analysis}

The data was collected from semi-structured interviews, which according to Silverman [40] and Walsham [41] provide rich data on respondents' interpretations of the problem under investigation. The unit of analysis was individual online shopping consumers. The sample was purposely selected, as participants must have had experience doing online shopping during the last three months and must be over the age of eighteen. The sample covered a wide range of demographics. It included twelve females and nine males ranging in age from twenty to sixty-five and with diverse educational backgrounds. All the participants had at least five years of familiarity using the internet and the majority used online shopping at least once or twice per month.

Based on extant literature and the research question of the study, a semi-structured interview guide was developed using several open-ended questions. Initially, the respondents were asked about their general use of, and experiences with, the internet and online shopping. Then questions were asked about privacy issues and how much these concerned them. After setting this background, several questions relating to psychological distance were asked of the participants (see Table 1). Twenty-one interviews were conducted with an interview duration ranging from 1545 minutes.

Table 1. Sample interview questions

\begin{tabular}{|l|l|}
\hline Topic & Sample Question \\
\hline $\begin{array}{l}\text { Internet Privacy } \\
\text { Concerns }\end{array}$ & $\begin{array}{l}\text { What is your idea about } \\
\text { the level of privacy on the } \\
\text { internet? }\end{array}$ \\
\hline Temporal Distance & $\begin{array}{l}\text { Is privacy an immediate } \\
\text { threat? }\end{array}$ \\
\hline Spatial Distance & $\begin{array}{l}\text { Compared to other } \\
\text { countries what is the level } \\
\text { of privacy in Australia? }\end{array}$ \\
\hline Social Distance & $\begin{array}{l}\text { Compared to others, how } \\
\text { likely are you to } \\
\text { experience a privacy } \\
\text { breach? }\end{array}$ \\
\hline Hypotheticality & $\begin{array}{l}\text { How likely are privacy } \\
\text { issues to occur in the } \\
\text { online shopping context? }\end{array}$ \\
\hline
\end{tabular}

We followed the content analysis method [40] to analyze the interview data. This method is useful to scrutinize content or contextual meaning of data through a systematic classification process of coding and by identifying themes or patterns. A directed content analysis method was considered more suitable as it allows for the identification of key concepts in an existing theory as initial coding categories [43]. The data was uploaded, the coding frame was developed using the CLT dimensions of psychological distance: spatial, temporal, social and hypothetical [11], and was coded using NVivo 11 software. A collaborative approach [44] based on discussion and consensus was followed by the research team to ensure reliability of the coding process. 


\section{Findings and discussion}

The qualitative analysis of the interview data revealed several insights of consumers' privacy concerns and psychological distant aspects. In the initial phase of the interview we discussed the level of awareness and concerns on privacy. The majority of the respondents were either highly concerned or to some extent concerned about their online privacy.

The feeling of distance between consumers and online retailers is well established in the literature [38, 39]. This disconnection that prevails in the virtual shopping environment transcends mere physical distance to create a psychological distance among the two parties [45]. This was evidenced by some of the comments made by the interview participants; "I feel disconnected to online versus physical world, I feel it's quite disconnected". Another participant mentioned, "I think because it's a virtual world, you are not actually seeing what goes on behind- where all that information is actually going and being sent. I think that is a disconnection".

This distance fosters privacy issues in the online environment, whereby according to one participant, "privacy is not an immediate issue and it is not even very tangible because it's online. It feels very distant to your personal physical world". These statements verify that the abstractness of privacy is multiplied in the more 'disconnected' online world.

The focus of this paper is to present how consumers' perceived aspects of psychological distance influence their privacy values, values received from online shopping, and privacy-related behavior. Figure 1 illustrates the frequency of codes that were identified in relation to the four main aspects of psychological distance. Out of 169 responses related to psychological distance, the majority expressed views about hypotheticality followed by spatial, social, and temporal distance aspects. Each of these aspects is discussed in detail in the following sections.

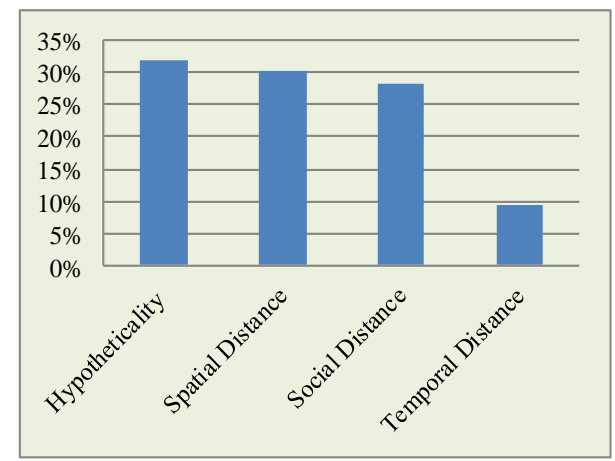

Figure 1. Frequency of top codes identified in the interview analysis

\subsection{Hypotheticality}

Respondents fundamentally characterized privacy in 'uncertainty' terms, which mostly arise from skepticism about the causes of privacy issues as well as its impacts. The majority of respondents agree that privacy is a real issue that exists in the current technology-mediated online space. However, some participants disparage privacy issues as being overly exaggerated by the media or society, which leads them to underestimate its probability. The majority fits with the claim that, "definitely there is an issue because even if it happens to one or two people, I would say that companies need to be very careful about it", but there are some respondents who believe, "although when you hear it on the news they often dramatize it as they do everything" or, "media blows everything, so it cannot be as large as the media says but seriously it [privacy] is a serious factor".

Prior CLT studies found that identifying events as unlikely or uncertain, leads events to be perceived as psychologically distant [32]. Especially in the online shopping context, the majority of participants believe privacy issues to be unlikely at individual level. One participant commented, "I think the risk of it [privacy breaches] would actually happening is quite low. You have to be reasonably unlucky for it to happen to you". Another mentioned, "it's like driving a car, you have to drive a car, you know you might crash or something like that, but still you have to do it". Especially, consumers believe, "you can get it [benefits] straight away, whereas the privacy things maybe, it's not for certain. You don't know that for sure". These responses of the participants and privacy literature both indicate that factors such as lack of privacy awareness and incomplete information could lead individuals to perceive privacy violations as unlikely [e.g., 46]. When the likelihood of an event is perceived as low, it encourages individuals to represent that event in highlevel abstract sense [32]. Therefore, perceiving privacy risks to be lower makes individuals feel them as hypothetically distant and abstract. This can lead privacy risks to appeal in more distant thinking but to be neglected in the immediate decision-making moment.

\subsection{Spatial distance}

Due to the abstract and intangible nature of privacy, it has been viewed psychologically distant. As declared by a participant, "it's not like you see somebody with a gun next to you, it's an unseen problem. So it's not like they are being reminded at every purchase". Given this abstract nature of privacy combined with the spatial 
disconnection in the online shopping environment, privacy becomes farther distant. Respondents viewed privacy issues as higher in online context when compared with the physical stores. One participant commented, "with a credit card, I'm giving it to a person over the counter. I see what they do with it. But someone who's got the details can always do something else when you can't see them", and another mentioned, "I generally have no idea of the person behind the shop [online]".

This situation is further heightened when consumers conduct online shopping with nonAustralian sellers. It was evident consumers do consider privacy to be safer when buying from Australia (psychologically closer), even when they do not know about any difference among sellers. One participant mentioned, "in my mind it is [privacy is safer when buying from Australia], I don't know if it really is, but in my mind it is". This was further validated by another participant saying, "relative to other countries I wouldn't know, but taking and educated guess... I like to think the Australian government is pretty good at putting out appropriate rules and regulations".

These findings align with the CLT contention that individuals form more abstract representations of the same phenomenon when the spatial distance is increased [30]. Therefore, it can be argued that, in the online environment, privacy becomes more abstract and distant in the mind of the consumers, making such values seem insignificant when decisions are made.

\subsection{Social distance}

CLT identifies distinctions among "self and other, similar and dissimilar others, familiar and unfamiliar others, in-group members and outgroup members, and status differences" as exemplars of social distance [35, p. 357]. As mentioned earlier, the uncertainty level and spatial abstractness of privacy make individuals to perceive privacy more distantly. This also leads individuals to believe the effects of privacy violations on them to be distant, which also makes them less worried in comparison with others. Hence, some respondents claimed, "it's unlikely I fall victim to someone who sells my information", and, "it's likely an attack might happen on anybody but I don't want to think of that happening to me [compared to others], so I would just say no [won't be affected]". Social distance is clearly reflected when participants compare themselves or their inner-group members with outsiders. For instance, a participant mentioned, "it [privacy violations] didn't happen to a lot of people [I know of]. My friends and family were not affected by it. If something happens to my friends or my family, if
I heard of something that happened, then I would be concerned".

Social distance is also largely reduced when individuals experience privacy breaches by self; "you know it happens, but because that's such an underground thing, it's almost not reality until something does happen to you". Individuals clearly distinguish the impact of privacy between themselves and others, and as implied by CLT, personal impacts of privacy are judged to be lower than social impacts [31]. This can bring consumers to ignore privacy concerns when actual decisions are made.

\subsection{Temporal distance}

CLT studies have found that individuals consider desirability over feasibility and central values such as privacy are more easily applied in distant-future thinking than in actual decision making [29]. Supporting these findings, our interview data also found that individuals put greater weight on values or benefits received from online shopping than on more abstract privacy values when it comes to actual purchasing and information disclosure. A participant shared, "you can see benefits more, but with privacy you won't be able to see it as long as it doesn't happen [and] even it happens it might take a longer time to happen". Similarly, claims such as, "you can get it [benefits] straight away, whereas the privacy things maybe, it's not for certain", clearly indicate the immediacy of shopping benefits and prominence of feasibility factors. It was interesting to note how individuals who had privacy breaches explained why they continue to shop online. According to one such individual, "I've had incidents of where security is being breached. It's little bit upsetting and shocking at the time but you come to terms with it". This indicates that personal experience of privacy breaches can sway consumers for a while, but as the time passes, privacy again becomes an abstract concept in the mind. Another dimension of temporal distance, related to the immediacy, was highlighted by the participants. Some individuals identified privacy to be "more of a concern in the past" or "a problem in the future" rather than an immediate threat in the current online context. As confirmed by the findings of Hallam and Zanella [19], we argue that temporally distant privacy values are undermined over more immediate benefits when behaviors are performed.

\subsection{Privacy paradox and psychological distance}


The analysis of psychological distance facades of online shopping consumers provides numerous insights to the privacy paradox. First, due to its abstract and superordinate nature, privacy is considered a higher mental construal. This mostly influences consumers' distant-future intentions and attitudes [34]. According to the participants, the intangibility and virtual nature of both online environment and privacy, with less likeliness of privacy risks, less immediacy of harm, and fewer personal experiences, make privacy a psychologically distant issue. When compared with privacy concerns, online shopping benefits are perceived as concrete, immediate and likely. As suggested by the CLT, when individuals are faced with value conflicts, secondary values such as benefits received from online shopping rise to prominence over central values such as privacy at the time of decisionmaking [35]. It can be argued that ideals and central values might not matter that much when on-the-spot decisions are made.

Online shopping thrives in the lives of today's consumers due to the convenience, competitive prices, high feasibility shopping experiences, and several other benefits. The tendency of individuals to consider feasibility rather than desirability in the psychologically proximal choices [29] gives the aforementioned benefits the lead over values for privacy. Also, consumers might have the desire to protect their privacy in the distant thinking, but the feasibility of such intention is tested when it comes to actual use of online services. Privacy paradox is ultimately the gap between privacy concerns and privacy behavior. It is clear that, consumers, at least to a certain level, value their privacy but fail to see that value when it comes to their actual decision making.

Based on the above findings and discussion, it is possible to ascertain that privacy paradox can be explained on the basis of how an individual construes privacy based on the perceived psychological distance.

\section{Discussion}

In this study, we examined the impact of different aspects of psychological distance on the construal of privacy values to enlighten the privacy paradox. As contended by the CLT, something can be psychologically distant to the extent that it is remote from our direct experience in time, or in space, when related to others, and unlikely to occur, and therefore construed abstractly [35]. Application of this theorem in the online shopping context revealed individuals' tendency to negate abstract and superordinate cognitive values of privacy over concrete and immediate affective values like gratification and convenience when actual behavior is performed.

Privacy paradox studies are predominantly directed by economic and social theories. For instance, Privacy Calculus Theory explains consumers based on a calculus thinking disclose information when benefits are perceived to outweigh risks of doing so [20]. However, behavioral economists highlight the influence of cognitive biases and heuristics beyond rationality when performing a certain behavior. Consistently with this view, we argue that calculus thinking is rather directed by the level of psychological distance or proximity of the concerned values (i.e. privacy or gratification) and how they are mentally construed.

The role of familiarity and trust on privacy decision making is well established in the literature [47]. Studies on CLT have found that increased familiarity decreases social distance and level of construal [35]. The interview participants also conveyed how familiarity, which also leads to trust, can help to reduce privacy concerns. Therefore, it is important that privacy decision making models consider how familiarity and trust would influence psychological distance on the construal of privacy concerns.

The findings of this study are important to privacy research in general. It is a common practice of privacy scholars to measure stated intentions (e.g., intention to disclose or transact) instead of actual behaviors [14]. This practice has been questioned by some scholars, claiming intentions are not always reflective of actual behaviors [7]. We support this argument by showing that intentions greatly suffer from distance biases. For example, individuals may well say that they will not disclose their information due to privacy concerns. But according to our findings such claims are largely influenced by temporal and hypothetical distances. Therefore, we suggest that future privacy research should capture or observe actual behaviors rather than solely relying on stated intentions.

Through this investigation, we provide several theoretical contributions. We uniquely contribute to the privacy scholarship by investigating multidimensional aspects of psychological distance to elucidate the privacy paradox. Also, by applying CLT, we contribute to the small volume of findings related to the cognitive and psychological aspects of decision making in IS literature [9]. The findings enrich CLT research by explicating the value-conflict between affective values of gratification and cognitive value of privacy using construal levels.

Our findings are also useful for ethics literature. Privacy paradox itself presents an ethical dilemma in which a central value such as privacy is underplayed in an individual's actual behavior. This sheds light on 
ethical decision making. Are consumers more influenced by the consequentialist views rather than deontological views in psychologically proximal situations? Apart from these contributions, by conducting a qualitative exploratory study we enrich both privacy and CLT literature which is currently dominated by survey and experimental methods of research.

\subsection{Limitations and future research}

While our study delivers several new insights, it is not without limitations. We conducted the research with a specific focus of understanding the impact of different aspects of psychological distance on privacy decision making. However, literature provides evidence of several cognitive biases and heuristics that can occur in conglomeration. For instance, studies on affect heuristic have found that consumers underestimate privacy risks when a positive effect is elicited [48]. Likewise, optimism bias influences individuals to believe they are at less risk when compared to others [49]. Such impacts either correspond with or influence the construal mechanisms in the mind. It is important that we identify the influence of other heuristics and biases on future CLT investigations.

CLT asserts that a main reason for psychologically distant objects to be construed at an abstract level is, this; moving away from immediate reality reduces the information we have about that object. For instance, we know little about something that happens somewhere else or sometime later compared with what we know of the here and now. Therefore, lack of information, knowledge, and awareness about privacy can lead to further abstract construal. We did not delve deeply into such aspects in the interview process, but future privacy and CLT explorations can greatly benefit from the findings of incomplete information and bounded rationality studies [46].

Other limitations include the general focus of the study in which we considered overall online shopping rather than a specific industry, specific technology effect (e.g., big data) or a platform (e.g., mobile devices). Also, we did not differentiate among types of information that can impact privacy differently based on the sensitivity. Future research avenues should consider these limitations. As in general qualitative research, our study can limit both inferences that can be drawn and the generalizability of findings [40]. Future research can thus greatly contribute to the privacy scholarship by reproducing this study using quantitative research methods with a larger sample size.

\subsection{Implications}

Privacy paradox can be considered a double-edged sword. Although consumers are concerned about privacy, unwillingness to act on those concerns can lead to further exploitation of personal data by the companies in the data-driven marketplace. In line with the findings of our study, consumers need to be aware that the abstract nature of privacy values can make them perceive privacy at a distant level. Therefore, this research informs consumers, companies, and policy makers about the significance of communicating the importance of privacy to make it more proximal, especially to consumers' online experiences. Information collecting companies should be ethical and responsible in informing users about the level of privacy in their websites and the importance of protecting personal information. The government policies and regulations could address the privacy paradox by altering current policies to protect consumer privacy and educate consumers to enhance knowledge and awareness of methods of protecting their privacy. Apart from that, privacy scholars should be informed that measurement of stated intentions greatly suffers from distance biases and therefore considering actual behaviors is more important and effective.

\section{Conclusion}

The majority believes in the importance of preserving their privacy. Yet, privacy issues appeal to individuals as psychologically distant; something which may or may not happen in the future, affecting distant places, and affecting people other than them. Directed by the Construal Level Theory, we identified that perceiving privacy as a distant value makes it an abstract phenomenon in people's minds. This leads to forming distant-future intentions or attitudes about privacy rather than to real action. Therefore, in answering the question; is privacy paradox a matter of psychological distance, we argue that, at least to a certain extent, psychological distance and construal level plays a significant role in explaining the privacy paradox. Our findings provide several contributions to theory and we also inform users, companies, and policy makers on their role to mitigate the psychological distance of privacy.

\section{References}

[1] R. Chakraborty, J. Lee, S. Bagchi-Sen et al., "Online Shopping Intention in the Context of Data Breach in Online 
Retail Stores: An Examination of Older and Younger Adults", Decision Support Systems, 89, 2016, pp. 47-56.

[2] B. Gupta, L.S. Iyer, and R.S. Weisskirch, "Facilitating Global E-commerce: A Comparison of Consumers' Willingness to Disclose Personal Information Online in the US and in India". Journal of Electronic Commerce Research, 11(1), 2010, pp. 41-52.

[3] T. Dinev, and P. Hart, "Internet Privacy Concerns and Social Awareness as Determinants of Intention to Transact", International Journal of Electronic Commerce, 10(2), 2006, pp. 7-29.

[4] G. Bansal, F.M. Zahedi, and D. Gefen, "Do Context and Personality Matter? Trust and Privacy Concerns in Disclosing Private Information Online". Information \& Management, 53(1), 2016, pp. 1-21.

[5] TRUSTe, The State of Online Privacy 2016, Available from: http://www.truste.com/blog/2016/01/28/state-onlineprivacy-2016/ [01 May 2017].

[6] Australian Government, Office of the Australian Information Commissioner, Australian Community Attitudes to Privacy Survey 2017, Available from: https://www.oaic.gov.au/engage-with-us/communityattitudes/australian-community-attitudes-to-privacy-survey$\underline{2017}$ [15 May 2017].

[7] P.A. Norberg, D.R. Horne, and D.A. Horne, "The Privacy Paradox: Personal Information Disclosure Intentions Versus Behaviors", Journal of Consumer Affairs, 41(1), 2007, pp. 100-126.

[8] T. Dinev, "Why Would We Care About Privacy?", European Journal of Information Systems, 23(2), 2014, pp. 97-102.

[9] S. Kokolakis, "Privacy Attitudes and Privacy Behaviour: A Review of Current Research on the Privacy Paradox Phenomenon". Computers \& Security, 64, 2017, pp. 122134.

[10] N. Liberman, and Y. Trope, "The Psychology of Transcending the Here and Now", Science, 322(5905), 2008, pp. 1201-1205.

[11] Y. Trope, and N. Liberman, "Construal-level Theory of Psychological Distance", Psychological Review, 117(2), 2010, pp. 440-463.

[12] Eyal, T., and N. Liberman, Morality and Psychological Distance: A Construal Level Theory Perspective, in: M. Mikulincer and P. Shaver (Eds.), The Social Psychology of Morality: Exploring the Causes of Good and Evil, American Psychological Association, Washington DC, 2012, pp. 185202.

[13] Australian Communications and Media Authority, Communications Report 2014-15, Available from:
http://www.acma.gov.au/theACMA/Library/Corporatelibrary/Corporate-publications/communications-report-201415 [01 May 2017].

[14] H.J. Smith, T. Dinev, and H. Xu, "Information Privacy Research: An Interdisciplinary Review", MIS Quarterly, 35(4), 2011, pp. 989-1016.

[15] F. Bélanger, and R.E. Crossler, "Privacy in the Digital Age: A Review of Information Privacy Research in Information Systems", MIS Quarterly, 35(4), 2011, pp. 10171042.

[16] M.R. Brown, and R. Muchira, "Investigating the Relationship between Internet Privacy Concerns and Online Purchase Behavior", Journal of Electronic Commerce Research, 5(1), 2004, pp. 62-70.

[17] S. Cockcroft, "Information Privacy: Culture, Legislation and User Attitudes", Australasian Journal of Information Systems, 14(1), 2006, pp. 55-68.

[18] J. Drennan, G. Sullivan, and J. Previte, "Privacy, Risk Perception, and Expert Online Behavior: An Exploratory Study of Household End Users", Journal of Organizational and End User Computing, 18(1), 2006, pp. 1-22.

[19] C. Hallam, and G. Zanella, "Online Self-disclosure: The Privacy Paradox Explained as a Temporally Discounted Balance between Concerns and Rewards", Computers in Human Behavior, 68, 2017, pp. 217-227.

[20] T. Dinev, and P. Hart, "An Extended Privacy Calculus Model for E-commerce Transactions", Information Systems Research, 17(1), 2006, pp. 61-80.

[21] H. Xu, X. Luo, J.M. Carrol et al., "The Personalization Privacy Paradox: An Exploratory Study of Decision Making Process for Location-aware Marketing", Decision Support Systems, 51(1), 2011, pp. 42-52.

[22] C. Lutz, and P. Strathoff, "Privacy Concerns and Online Behavior - Not So Paradoxical After All? Viewing the Privacy Paradox through Different Theoretical Lenses", Working Paper, 15 April 2014, Available from: http://dx.doi.org/10.2139/ssrn.2425132 [01 May 2017].

[23] S. Utz, and N.C. Krämer, "The Privacy Paradox on Social Network Sites Revisited: The Role of Individual Characteristics and Group Norms", Cyberpsychology: Journal of Psychosocial Research on Cyberspace, 3(2), 2009, pp. 1-10.

[24] H. Cho, J.S. Lee, and S. Chung, "Optimistic Bias about Online Privacy Risks: Testing the Moderating Effects of Perceived Controllability and Prior Experience", Computers in Human Behavior, 26(5), 2010, pp. 987-995.

[25] C. Jensen, C. Potts, and C. Jensen, "Privacy Practices of Internet Users: Self-reports Versus Observed Behavior", 
International Journal of Human-Computer Studies, 63(1), 2005, pp. 203-227.

[26] R. Wakefield, "The Influence of User Affect in Online Information Disclosure", The Journal of Strategic Information Systems, 22(2), 2013, pp. 157-174.

[27] A. Acquisti, and J. Grossklags, "Losses, Gains, and Hyperbolic Discounting: An Experimental Approach to Information Security Attitudes and Behavior", 2nd Annual Workshop on Economics and Information Security-WEIS, Maryland, USA, 2003.

[28] Y.M. Baek, "Solving the Privacy Paradox: A Counterargument Experimental Approach", Computers in Human Behavior, 38, 2014, pp. 33-42.

[29] N. Liberman, and Y. Trope, "The Role of Feasibility and Desirability Considerations in Near and Distant Future Decisions: A Test of Temporal Construal Theory", Journal of Personality and Social Psychology, 75(1), 1998, pp. 5-18.

[30] K. Fujita, M.D. Henderson, J. Eng et al., "Spatial Distance and Mental Construal of Social Events", Psychological Science, 17(4), 2006, pp. 278-282.

[31] I. Liviatan, Y. Trope, and N. Liberman, "Interpersonal Similarity as a Social Distance Dimension: Implications for Perception of Others' Actions", Journal of Experimental Social Psychology, 44(5), 2008, pp. 1256-1269.

[32] C. J. Wakslak, Y. Trope, N. Liberman et al., "Seeing the Forest when Entry is Unlikely: Probability and the Mental Representation of Events", Journal of Experimental Psychology: General, 135(4), 2006, pp. 641-653.

[33] T. Eyal, N. Liberman, and Y. Trope, "Judging Near and Distant Virtue and Vice", Journal of Experimental Social Psychology, 44(4), 2008, pp. 1204-1209.

[34] A. Ledgerwood, Y. Trope, and S. Chaiken, "Flexibility Now, Consistency Later: Psychological Distance and Construal Shape Evaluative Responding", Journal of Personality and Social Psychology, 99(1), 2010, pp. 32-51.

[35] Liberman, N., Y. Trope, and E. Stephan, Psychological Distance, in: A. Kruglanski and E. Higgins (Eds.), Social Psychology: Handbook of Basic Principles, Guilford Press, New York, 2007, pp. 353-383.

[36] D. Read, and G. Loewenstein, "Time and Decision: Introduction to the Special Issue", Journal of Behavioral Decision Making, 13(2), 2000, pp. 141-144.

[37] D.H. Kim, Y.H. Sung, S. Y. Lee et al., "Are You on Timeline or News Feed? The Roles of Facebook Pages and Construal Level in Increasing Ad Effectiveness", Computers in Human Behavior, 57, 2016, pp. 312-320.
[38] N. Hartley, and T. Green, "Consumer Construal of Separation in Virtual Services", Journal of Service Theory and Practice, 27(2), 2017, pp. 358-383.

[39] P.R. Darke, M.K. Brady, R.L. Benedicktus et al., "Feeling Close from Afar: The Role of Psychological Distance in Offsetting Distrust in Unfamiliar Online Retailers", Journal of Retailing, 92(3), 2016, pp. 287-299.

[40] Silverman, D., Interpreting Qualitative Data: A Guide to the Principles of Qualitative Research, Sage, London, 4th ed., 2011.

[41] G. Walsham, "Interpretive Case Studies in IS Research: Nature and Method", European Journal of Information Systems, 4(2), 1995, pp. 74-81.

[42] K. Conboy, G. Fitzgerald, and L. Mathiassen, "Qualitative Methods Research in Information Systems: Motivations, Themes, and Contributions", European Journal of Information Systems, 21(2), 2012, pp. 113-118.

[43] W.J. Potter, and D. Levine-Donnerstein, "Rethinking Validity and Reliability in Content Analysis", Journal of Applied Communication Research, 27, 1999, pp. 258-284.

[44] P. Smagorinsky, "The Method Section as Conceptual Epicenter in Constructing Social Science Research Reports", Written Communication, 25(3), 2008, pp. 389-411.

[45] S.M. Edwards, J.K. Lee, and C.L. Ferle, "Does Place Matter when Shopping Online? Perceptions of Similarity and Familiarity as Indicators of Psychological Distance", Journal of Interactive Advertising, 10(1), 2009, pp. 35-50.

[46] A. Acquisti, and J. Grossklags, "Privacy and Rationality in Individual Decision Making", IEEE Security \& Privacy, 3(1), 2005, pp. 26-33.

[47] D.J. Kim, D.L. Ferrin, \& H.R. Rao, "A Trust-based Consumer Decision-making Model in Electronic Commerce: The Role of Trust, Perceived Risk, and Their Antecedents", Decision Support Systems, 44(2), 2008, pp. 544-564.

[48] F. Kehr, T. Kowatsch, D. Wentzel et al., "Blissfully Ignorant: The Effects of General Privacy Concerns, General Institutional Trust, and Affect in the Privacy Calculus", Information Systems Journal, 25(6), 2015, pp. 607-635.

[49] Y.M. Baek, E. Kim, and Y. Bae, "My Privacy is Okay, but Theirs is Endangered: Why Comparative Optimism Matters in Online Privacy Concerns", Computers in Human Behavior, 31, 2014, pp. 48-56. 